\title{
GAP-ANALYTICS (GEOLOCATION AND PLACES-ANALYTICS)
}

\author{
Yogesh R. Jadhav', Sunilkumar R.Yadav ${ }^{2}$, Gautam J. Kale ${ }^{3}$, Atul R. Barve ${ }^{4}$ \\ ${ }^{1,2,3,4}$ Student- Sanghavi College of Engineering, Nashik
}

\begin{abstract}
World is the full of interesting places and can be confusing and overwhelming at the same time. There can be instances where people might get lost or enter unsafe areas unknowingly. To address such issue, GAP-Analytics (Geolocation and PlaceAnalytics) is an android app and a Web application that helps its users to know their current location, search for places of their interest like hotels, police-station, hospitals etc. around them. Using android as its platform is beneficial looking at the market share of android OS as it can reach out many people. It will help data analysts to get statistics of activity of people around a geographic location from online dash board by maintaining the privacy of the users. Smart phones are great devices which are intelligent and if used properly, it can save us from a lot of unpleasant situations. By using location services from android environment, this app provides location features. This app uses Google location API V2, Google Places API and Google Maps api in its android client application. On the server side it uses windows ASP.NET Web Services to serve client requests.
\end{abstract}

Key Words: LBS, Geolocation, Geofencing, GPS, API, WCF, REST. $* * *$

\section{INTRODUCTION}

Location Based Services (LBS) is an area of services which focuses on providing a custom regional experience to the users by providing them information and services according to their present location. GAP-Analytics is the location based application which includes an android app as its client and .NET Web service as its server component. Android app provides current location of the user and thus, provides location input to the system to provide various location based services like tracking, geofencing, finding points of interests, finding who is around you and many other features.Location based services have evolved over time and has become very vital in current digitized world. Internet is spreading in every nook and corner of the world at a very brisk pace. Along with internet, gadgets like smart phones have changed the way we live and work in today's world. The reasons for location services to become a key player in general people's life are:

1. High end processing power of smart phones

2. High speed internet connectivity

3. Cheap manufacturing cost of GPS hardware

4. General interest of the consumer in gadgets

Location based services (LBS) are on the rise with advancement in technology and low cost of integration of GPS on mobile phones. Users can enjoy regional services and get to know about their surroundings far more easily than ever before. Family safety and security and be made easy by sharing location among family members. World is digitizing, so it is necessary to get the maximum juice out of it and use it for simplifying and enhancing our lives. With technology as a part of our life, we have a bright future and can lead intelligent life by outsourcing mundane tasks to technology. This project will try to provide travel and safety utility to its users. This project will try to deal with many aspects of LBS and will try to provide a good user experience and helpful features that will surely help the user and save his time.

\section{ARCHITECTURE}

This app is based on client server model where android app is the client whereas ASP.Net web service serves client from the server. Server gets the requesting client the coordinates of the subject to be tracked through HTTP request and response method. We can use either SOAP or REST full service model for client-server communication implementation.

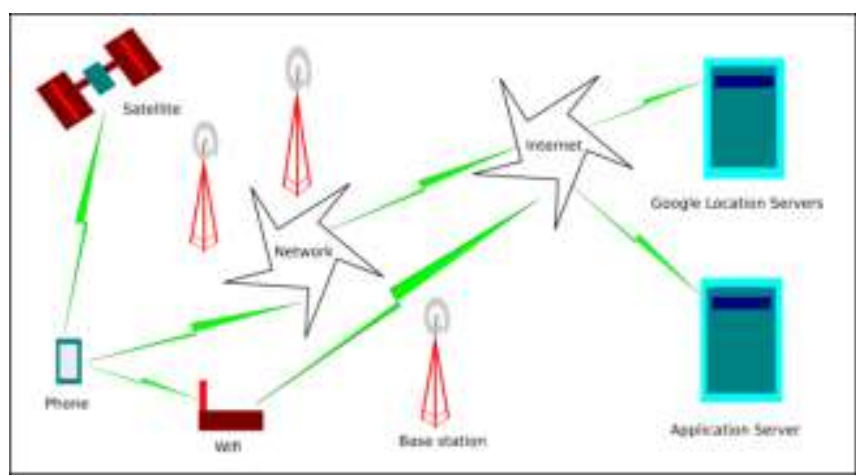

Fig. 1: Architecture

Our project works on the principle of distributed computing. In distributed computing, the system is divided into components which are physically remote to each other. But when user uses this system, he gets the illusion that the computer he is using has all its resources at one place.

In this project, the components of distributed system are:

1. Android Client: This is the app that is installed on end user's smart phone.

2. Web server: It provides back end for storing location related data.

Whenever client starts his app, he can use the data available on servers without knowing that such data is stored remotely and is being transferred through internet. Such distributed 
nature is seen when a user tracks another user, searches for nearby places and checks if anyone is around him or not.

Application specific server stores user location data on the cloud and acts as an interface between multiple android clients. Server performs different operations like computing distance between two locations, searching contacts, registering users, sending alerts and geofencing. For all data relating to nearby places and maps, it is handled by Google servers by the use of Location service APIs from Google. To get detailed information about your current location, wikipedia provides the information. We make use of GPS, Wifi, network signal and Proximity sensor for their respective operations.

We use REST service framework to make calls to different servers from our client app.

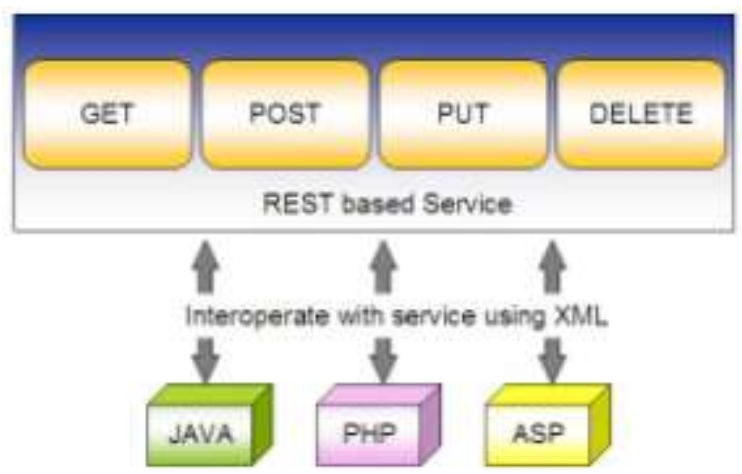

Fig. 2: REST framework

- REST is an architectural style for networked hypermedia applications, it is primarily used to build Web services that are lightweight, maintainable, and scalable.
- REST does not put a restriction on the format of a representation.

- It is platform independent, any applicatio can access REST services.

- It is the most widely accepted framework for recent application development

\subsection{Algorithm}

Input: Current location, proximity sensor reading.

Output: Location aware service like profile automation, reminder, important places around you, weather, tracking other users, proximity alert.

Step 1: Install android client app.

Step 2: Register using email, name and mobile number.

Step 3: Login Step

Step 4: From options menu select operation you want to perform:

- My Location: Select this option to get your current location

- Add Contact: Manually add contact to this app.

- Contacts: View list of contacts with which you are sharing location.

- Places: Find interesting and important places around you.

- Geofences: Configure your geofences to automate tasks or get alerts.

- My Location Wiki: Opens a wikipedia link to your current location and gives details.

- Forecast: Get weather forecast.

Step 5: Stop

\subsection{State Transition Diagram}

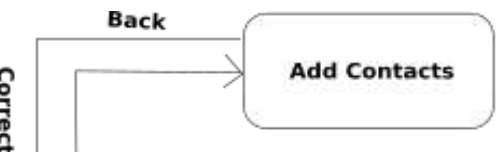

Option Add
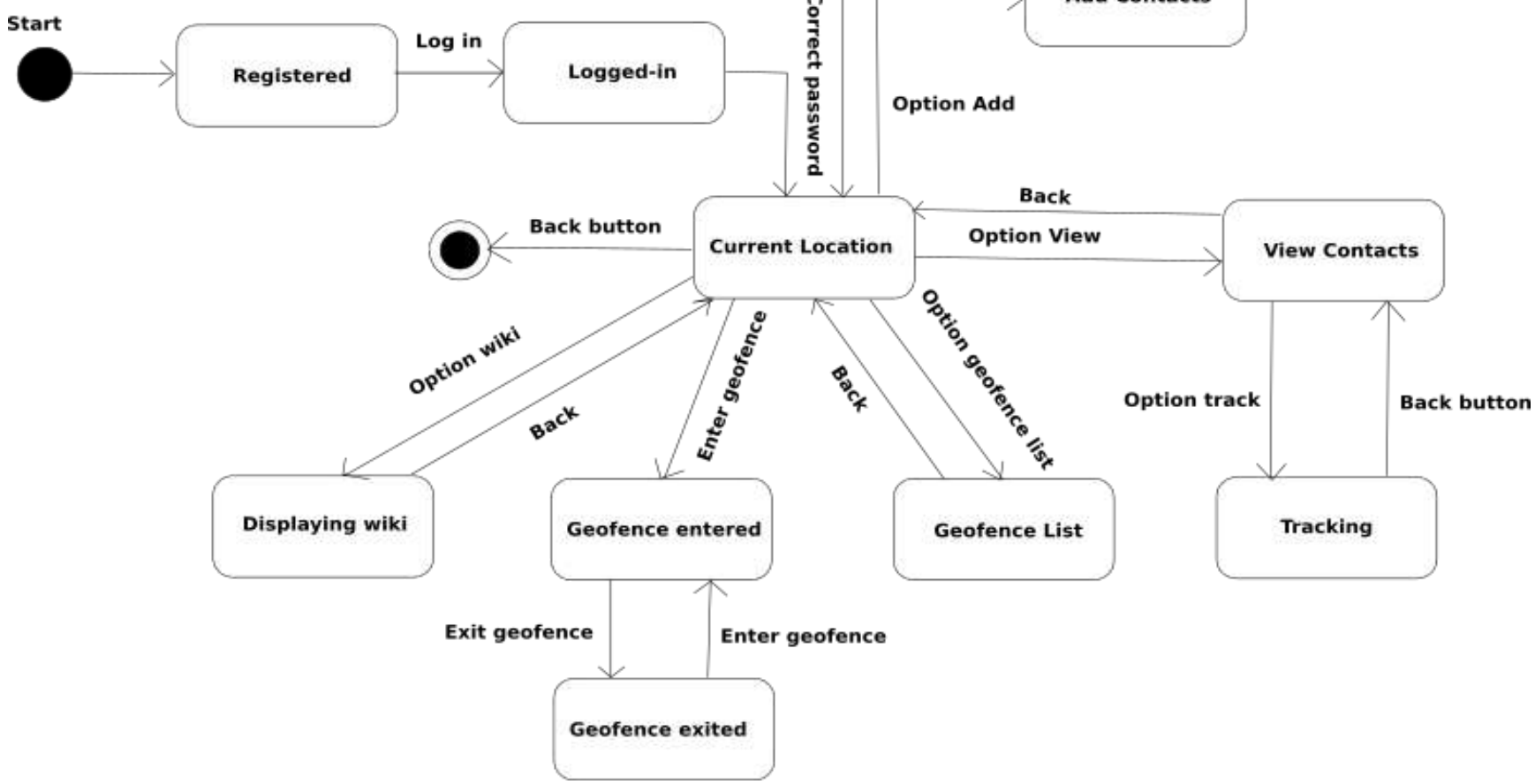

Fig. 3: State transition diagram 
State Transition example shows the state transition diagram of android app. The states are represented in ovals and state of system gets changed when certain events occur. The transitions from one state to the other are represented by arrows. The Figure shows important states and events that occur while running the android app.

\subsection{System Description}

Let $\mathrm{G}$ represent from system as a set of tuples as follows:

$\mathrm{G}=\mathrm{I}, \mathrm{O}, \mathrm{T}, \mathrm{S}, \mathrm{W}$

where, $\mathrm{I}=$ Input $\mathrm{O}=$ Output $\mathrm{T}=$ Time $\mathrm{S}=$ Sensors $\mathrm{W}=$ Web service

$\mathrm{I}=$ Input $=\mathrm{L}$ where, $\mathrm{L}=$ Location $=$ (latitude, longitude)

$\mathrm{O}=$ Output $=\mathrm{L}, \mathrm{W}, \mathrm{P}, \mathrm{F}$

where,

L=Location, $\mathrm{W}=$ Weather, $\mathrm{P}=$ Places $\mathrm{F}=$ Geofence alerts

$\mathrm{S}=$ Sensors $=$ GPS, Wifi, Proximity $\mathrm{W}=$ Web service $=\mathrm{L} 1$, S1, P1 where,

L1=Load operation $\mathrm{S} 1=$ Store Operation $\mathrm{P} 1=$ Processing data

$\mathrm{T}=\mathrm{Time}=\mathrm{R} 1+\mathrm{R} 2$

where, R1 = Request, R2 = Response

$\mathrm{R} 1$ = Gathering input (t1) + Transport on internet (t2) +

Server (t3) + Weather (t4) + Google (t5)

$\mathrm{R} 2=$ Google $(\mathrm{t} 1)+$ Weather $(\mathrm{t} 2)+$ Server $(\mathrm{t} 3)+$ Transport $(\mathrm{t} 4)$

+ Formatting received output (t5)

$\mathrm{R} 1=\mathrm{ti}=\mathrm{t} 1+\mathrm{t} 2+\mathrm{t} 3+\mathrm{t} 4+\mathrm{t} 5$

$\mathrm{R} 2=\mathrm{tj}=\mathrm{t} 1+\mathrm{t} 2+\mathrm{t} 3+\mathrm{t} 4+\mathrm{t} 5$

$\mathrm{T}=\mathrm{R} 1+\mathrm{R} 2$

Thus, $\mathrm{T}$ denotes total time taken for processing of input and giving out the output.

Distance between two coordinates

Haversine formula:

$\mathrm{a}=\sin 2(4 \varphi / 2)+\cos \varphi 1 \cdot \cos \varphi 2 \cdot \sin 2(4 \lambda / 2)$

$\mathrm{c}=2 \cdot \operatorname{atan} 2(\mathrm{a},(1 \mathrm{a}))$

$\mathrm{d}=\mathrm{R} \cdot \mathrm{c}$

where $\varphi$ is latitude, $\lambda$ is longitude, $\mathrm{R}$ is earths radius (mean radius $=6,371 \mathrm{~km}$ ); note that angles need to be in radians to pass to trig functions!

Thus, working of our algorithm takes fixed linear time which is dependent on numbers of inputs given to our algorithm.

\subsection{System Features}

\section{Tracking}

- Find your friends/family/colleagues on Google Map.

- Find who around you within a specified range.

- Find last known location.

- Send distress message/alarm to your near ones.

\section{Geofencing}

It allows user of the system to draw zones around place of work, customer sites and secure areas these geofences when crossed by an equipped person can trigger a warning to the user via SMS or email.

- Detect if you enter/exit an area of interest/danger.
- Set alarm/reminder for a particular location.

- Geofencing used with child location services, can notify parent if a child leaves a designated area.

- Change mobile profile depending on particular geofence

\section{Places}

- Find police station around you.

- Find the Bus stop/air-ports around you.

- Find hotels near you.

- Find hospitals around you.

- Find ATMs near you.

- Find the important landmarks near you.

4. Weather

- Get current weather based on your current location.

- Get current weather of your family/friends/colleagues from their location.

\section{Analytics}

- Get data about people's location patterns over a period of time.

- Get danger zone areas from users who triggered SOS messages.

- Get report on frequently visited places by users.

\subsection{Important Classes}

Table: Important Classes

\begin{tabular}{|c|c|}
\hline Class & Description \\
\hline Activity/ActionBarActivity & $\begin{array}{l}\text { Provides activity life-cycle } \\
\text { methods to } \\
\text { manage activity operation. }\end{array}$ \\
\hline LocationListener & $\begin{array}{l}\text { Provides facility to get cur- } \\
\text { rent location } \\
\text { from an android device. }\end{array}$ \\
\hline GoogleApiClient & $\begin{array}{l}\text { Provides facility to connect } \\
\text { to Google } \\
\text { Play Services and get loca- } \\
\text { tion updates. }\end{array}$ \\
\hline GoogleMap & $\begin{array}{l}\text { Provides facility to integrate } \\
\text { and manipulate Google Map } \\
\text { in our app. }\end{array}$ \\
\hline Service & $\begin{array}{l}\text { Allows to create background } \\
\text { service } \\
\text { which is isolated from main } \\
\text { app process. }\end{array}$ \\
\hline BroadcastReceiver & $\begin{array}{l}\text { Provides facility to listen for } \\
\text { different } \\
\text { broadcasts (events) arising } \\
\text { within Android OS. }\end{array}$ \\
\hline
\end{tabular}




\subsection{APIs}

\section{Google's Location API V2:}

This is the API provided by Google to get access to location data from android system through API calls. These API calls interact with android OS and GPS hardware to get current location of the device. Users need Google Play Services installed to get service from this API. Developers also need to obtain API key from Google Developer Console. Depending upon the usage, we need to get subscription from Google to use their location API. Google now provides FusedLocation API which is energy efficient and intelligently chooses between available location providers such as network, Wifi and GPS to determine current location.

\section{OpenWeatherMap API (weather):}

OpenWeatherMap API is open source weather API based on Creative Commons 2 license which provides weather updates covering the whole world. It is simple to use and provides with required weather data for the given location coordinates. This project aims to provides location based experience by combining weather data to help users get to know more about a particular location. In order to use this API, we need to obtain API key which will identify our application requests to its servers.

\section{LEGAL REQUIREMENTS}

- Need developer key to upload app to Play Store. Need to obtain API key to use Google Location API V2.

- To use Location API for enterprise level usage, we need a legal license from Google which can be purchased online from their website.

- User should allow location service and should not use this app if user finds it is invading their privacy. Aim of this app is to provide location based experience with safety, thus you should choose carefully the people (trusted) with whom you want to share location.

- It is illegal to use Google location data on maps other than Google maps. You also cannot cache maps from Google maps without permission.

- We need to obtain free API key from OpenWe therMap to use their weather service.

- Maintaining privacy is important requirement which can be achieved through encryption.

\section{USER PROFILES}

4.1 Registered User: This is the user who installed our app and registered for using location based services. This user can perform following actions:

- Tracking

- Geofencing

- Find who is around you

- Get weather report of current location and location of tracked users

- Get detailed information about current location through wiki page
4.2 New User: This is a user who has installed our app but haven't yet registered to use our app to avail location based services. This user can at his status can perform following actions:

- Install our app

- Register to use our app

4.3 Admin: This user can view all the registered users and can track registered users in the case of emergency and emergency requests from other registered users. He can perform following actions:

- Track any user in case of emergency

- Delete accounts of any registered user

- Sends emergency announcements to registered users.

4.4 Data Analyst: This is the user who deals with analysis of large amount of data. In our project, such analysts can study location patterns and identify danger zones based on user's emergency message origins. His actions are as follows:

- Find location patterns for their interest

- Get data about danger zones from user alerts

- He can predict advertising strategy based on location of users

\section{OPERATIVE ENVIRONMENT}

Android Operating System: Android is an open source operating system maintained by Google and is quite popular among smart phone community. Developing in android requires knowledge about Java programming language. Android runs on embedded SoC which includes GPS, Wifi, Processor, RAM, USB interface etc.

Web Service: Web service runs on cloud environment with shared hosting companies like Godaddy which charge you to use their resources to run your web service. As the hardware used is of hosting provider, developer do not need to invest in high cost hardware.

\section{PROJECT RESOURCES}

Hardware resources: Android smart phone, cloud web server host

Software resources: Google Location API, OpenWeatherMap API, Android Studio IDE, Visual Web Developer IDE, Project Management tool-Wrike, Wikipedia

Human Resources: Android developer, ASP.NET developer, tester, Documentation engineer

\section{CONCLUSION}

Users of this app can be sure about the movement of their near and dear ones. People can track and get valuable information about their surrounding environment through Google Places API. We can make proper use of smart phones as an intelligent device by using its features like wifi, mobile network data and GPS. Thus, city planners, analysts and security services can use this project to study location patterns and monitor people about their geo-points of interest. 


\section{ACKNOWLEDGMENT}

The authors wish to thank Prof. Bajirao S. Shirole (Sanghavi college of engineering, nashik) for providing guidance and proper direction in developing our project and necessary motivation and support.

\section{REFERENCES}

[1] J Schiller, A Voisard, Location-based services, Publisher: Morgan Kaufman, Year: 2004, 500 Sansome Street, Suite 400, San Francisco, CA 94111

[2] Android Wireless Application Development by Shane Condor and Lauren Darcy

[3] OpenWeatherMap

http://www.openweathermap.org/api

[4] Google Location API https://www.developers.google.com/maps/documentatio n/android-api/

[5] Geoawsomeness http://www.geoawesomeness.com/knowledge-base/ location-based-services/location-based-services-chronology/

[6] Google Developer Console https://console.developers.google.com/project

[7] Android tutorial https://developer.android.com/training/index.html 